\title{
Breakfast characteristics and its association with daily micronutrients intake - A systematic review and meta-analysis
}

\author{
Natalia Giménez-Legarre $^{1,2}$, María Luisa Miguel-Berges ${ }^{1,2}$, Paloma Flores-Barrantes ${ }^{1,2}$, \\ Alba María Santaliestra-Pasías ${ }^{1,2}$ and Luis A Moreno ${ }^{1,2}$ \\ ${ }^{1}$ GENUD (Growth, Exercise, Nutrition and Development) Research Group, Facultad de Ciencias de la Salud, \\ Universidad de Zaragoza, Zaragoza, Spain and \\ ${ }^{2}$ Instituto Agroalimentario de Aragón (IA2), Instituto de Investigación Sanitaria Aragón (IIS Aragón), Zaragoza, \\ Spain
}

\section{Abstract}

Introduction: Breakfast (BF) is widely recognized as an important component of a healthy lifestyle and represents an important source of key nutrients in the diet for both adults and children. Furthermore, BF consumption seems to be associated with a better intake of vitamins and minerals in both, children and adolescents.

The aim of this study was to investigate the associations between frequency and characteristics of BF consumption and its relation with micronutrients intake.

Material and Methods: An exhaustive search was carried out in three datasets in March 2019. The search strategy used to identify the articles was as follow: breakfast, food beverages appetite regulation, child nutritional physiological phenomena, diet, digestion, eating, feeding behavior, gastrointestinal absorption, hunger, nutritional requirements, nutritional status, nutritive value, breakfast skipping, meal skipping, fasting, food preferences, diet therapy, child, preschool, adolescent, breakfast skipping and meal skipping. Two independent reviewers performed the data extraction and assessed their quality and risk of bias following the PRISMA methodology and using the Cochrane risk of bias tool. Meta-analysis was performed comparing results based on type of BF: skip BF, Ready To Eat Cereal (RTEC) BF and other types of BF. DerSimonian and Laird estimators using random effects models were applied for continuous data. Effect sizes were calculated for each outcome.

Results: Out of 3105 articles, we selected 30 full-text articles for inclusion and 7 were considered for meta-analysis.. Children who usually skip BF had significantly lower daily intake of vitamin A (SMD, -10.407; 95\%CI: -14.147, -6.667) and vitamin C (SMD, -4.127 ; 95\%CI: $-5.091,-3.162$ ) than BF consumers. The intake of vitamin B1 (SMD, -16.378; 95\%CI: -29.110, -3.647) and vitamin B2 [SMD, -14.757; 95\%CI: -20.247, -9.268] was lower in skippers than RTEC BF consumers.

Regarding to minerals, children who usually consume BF had significantly higher daily consumption of Calcium (SMD, -7.034; 95\%CI: -9.029, -5.040), Iron (SMD, -6.552; 95\%CI: -9.242, -3.861) and Sodium (SMD, -3.395; 95\%CI: -5.554, -1.236) than BF skippers. The intake of Magnesium (SMD, -10.903; 95\%CI: -18.078, -3.729) and Potassium (SMD, -6.972; 95\%CI: -10.689, -3.254) was higher in RTEC BF consumers than BF skippers.

Discussion: Evidence suggested that BF consumption and RTEC breakfast consumption seems to be associated with better micronutrient intake

\section{Conflict of Interest}

There is no conflict of interest 